\title{
Testing for Identification in SVAR-GARCH Models
}

\author{
Helmut Luetkepohl* \\ George Milunovich**
}

* DIW Berlin and Freie Universitaet Berlin, Germany ** Macquarie University, Australia

This research was supported by the Deutsche Forschungsgemeinschaft through the SFB 649 "Economic Risk".

http://sfb649.wiwi.hu-berlin.de ISSN 1860-5664 


\title{
Testing for Identification in SVAR-GARCH Models!
}

\author{
Helmut Lütkepohl \\ DIW Berlin and Freie Universität Berlin, Mohrenstr. 58, 10117 Berlin, \\ Germany \\ email: hluetkepohl@diw.de \\ and \\ George Milunovich \\ Macquarie University, Sydney, NSW, 2109, Australia \\ email: george.milunovich@mq.edu.au
}

May 26, 2015

\begin{abstract}
Changes in residual volatility in vector autoregressive (VAR) models can be used for identifying structural shocks in a structural VAR analysis. Testable conditions are given for full identification for the case where the volatility changes can be modelled by a multivariate GARCH process. Formal statistical tests are presented for identification and their small sample properties are investigated via a Monte Carlo study. The tests are applied to investigate the validity of the identification conditions in a study of the effects of U.S. monetary policy on exchange rates. It is found that the data do not support full identification in most of the models considered, and the implied problems for the interpretation of the results are discussed.
\end{abstract}

Key Words: Structural vector autoregression, conditional heteroskedasticity, GARCH, identification via heteroskedasticity

JEL classification: C32

\footnotetext{
${ }^{1}$ We thank Aleksei Netšunajev, Minxian Yang, Till Strohsal, and Adrian Pagan for helpful comments on an earlier version of this paper. The paper was written while the first author was a Bundesbank Professor at the Freie Universität Berlin. Part of the research was carried out while he was visiting Macquarie University, Sydney, and parts were done while the second author was visiting the Freie Universität Berlin. Financial support was provided by the Deutsche Forschungsgemeinschaft through the SFB 649 "Economic Risk". Milunovich also acknowledges support from Australian Research Council Discovery Grant DP120102239.
} 


\section{Introduction}

In structural vector autoregressive (SVAR) analysis, identifying the structural shocks properly is typically one of the main problems. Several proposals have been made on how to proceed in specifying the shocks since the publication of the seminal article by Sims (1980) who argued convincingly in favour of the VAR approach. Recently it has been suggested that changes in volatility may be used for identification in SVAR models. For example, Rigobon (2003) and Lanne and Lütkepohl (2008) use changes in the unconditional variance of the reduced form residuals for identification while Normandin and Phaneuf (2004), Bouakez and Normandin (2010), and Lanne, Lütkepohl and Maciejowska (2010) take advantage of conditional heteroskedasticity in this context.

Conditional heteroskedasticity can be modelled in different ways. Whereas Lanne et al. (2010) assume that it is driven by a Markov process, Normandin and Phaneuf (2004), Bouakez and Normandin (2010) and a number of other authors use multivariate generalized autoregressive conditional heteroskedasticity (GARCH) processes. For these processes formal conditions for identification are available (see Sentana and Fiorentini (2001) and Milunovich and Yang (2013)). In practice it is not straightforward to check these conditions, as they are expressed in terms of true/population quantities while in reality we observe their estimated counterparts. An additional complication results from the fact that under the null hypothesis the model is unidentified, which rules out tests based on estimated structural coefficients. Therefore the standard approach in applied work is to use informal checks. In this paper we argue that in particular in macroeconometric studies it cannot be taken for granted that the identification conditions are satisfied and therefore informal arguments may not be sufficient to actually ascertain identification of the model. We point out, that formal statistical tests have to be used for investigating identification and argue that the tests proposed by Lanne and Saikkonen (2007) can be used for that purpose in the current SVAR setting. We also construct an alternative test that can be used in this context. We explore the small sample size and power properties of the alternative tests when applied in the present situation. Given the relatively low power of the tests in the present context, we discuss testing strategies that are informative on the issue of interest here, namely the identification of the structural shocks.

The study makes the following contributions. First, we present the model setup and clarify the identification conditions in such a way that we can use the Lanne-Saikkonen and related tests for the present purpose. Second, we provide three alternative tests that may be used to test the sufficient identifi- 
cation condition in the SVAR-GARCH context. We explore the small sample properties of the tests in a Monte Carlo simulation experiment and derive strategies for testing for identification in SVAR-GARCH models. Third, we use these strategies in an empirical application investigating the importance of U.S. monetary policy shocks for exchange rates. It is based on a study by Bouakez and Normandin (2010) who also use the SVAR-GARCH approach in their analysis but do not perform formal statistical tests for identification of their shocks. We argue that there are no compelling reasons for taking identification for granted in this particular framework and we present evidence that in some of the models used by Bouakez and Normandin (2010) it is indeed not supported by the data. We discuss the implications of these findings for the effects of monetary policy on exchange rates.

The study is structured as follows. In Section 2 the model setup is presented and the formal identification conditions are stated. The statistical tests for identification are discussed in Section 3 and the Monte Carlo experiment investigating their small sample properties is reported in Section 4. The empirical application is discussed in Section 5 and conclusions follow in Section 6.

\section{The Model}

\subsection{Reduced Form and Structural Form}

The reduced form of our model is a $K$-dimensional vector autoregressive (VAR) process,

$$
y_{t}=\nu+\Pi_{1} y_{t-1}+\cdots+\Pi_{p} y_{t-p}+u_{t},
$$

where $\nu$ is a $K$-dimensional constant term, the $\Pi_{j}(j=1, \ldots, p)$ are $(K \times K)$ coefficient matrices and $u_{t}$ is the serially uncorrelated error term with mean zero and unconditional covariance matrix $\Sigma_{u}$.

The structural errors, denoted by $\varepsilon_{t}$, are obtained by a linear transformation from $u_{t}$,

$$
\varepsilon_{t}=B^{-1} u_{t} \quad \text { or } \quad u_{t}=B \varepsilon_{t} .
$$

Of course, the structural errors are also white noise and, hence, serially uncorrelated. In addition, they are assumed to be instantaneously uncorrelated and their variances are standardized to unity, that is, $\varepsilon_{t} \sim\left(0, I_{K}\right)$. Consequently, the transformation matrix $B$ must be such that $B B^{\prime}=\Sigma_{u}$.

The $B$ matrix is the matrix of instantaneous effects of the structural shocks on the observed variables $y_{t}$. Uniqueness of the $B$ matrix and, hence, 
identification of the shocks is often ensured by zero restrictions on $B$ that amount to specifying that specific shocks have only a delayed effect on some of the variables. Such restrictions are quite common in a conventional SVAR analysis. Alternatively, exclusion restrictions on the instantaneous relations between the variables are sometimes imposed on $B^{-1}$ in order to ensure uniqueness of the matrix $B$. Restrictions on the long-run effects of the shocks are also frequently used for identification. In any case, a uniquely identified model requires a sufficient set of restrictions on $B$.

In structural VAR analysis the identifying restrictions are typically kept to a minimum and are, hence, just-identifying at best. In that case they cannot be tested against the data. In the following we take advantage of conditional heteroskedasticity to obtain a unique $B$ matrix or at least get some additional identifying information that can be helpful in assessing conventional identifying restrictions.

\subsection{Identification via GARCH}

Suppose $u_{t}$ has a GARCH structure so that

$$
u_{t}=B\left[\begin{array}{cc}
\Lambda_{t \mid t-1}^{1 / 2} & 0 \\
0 & I_{K-r}
\end{array}\right] e_{t},
$$

where the $e_{t}$ are independently, identically distributed, i.e., $e_{t} \sim \operatorname{iid}\left(0, I_{K}\right)$, and

$$
\Lambda_{t \mid t-1}=\left[\begin{array}{ccc}
\sigma_{1, t \mid t-1}^{2} & & 0 \\
& \ddots & \\
0 & & \sigma_{r, t \mid t-1}^{2}
\end{array}\right]
$$

is an $(r \times r)$ diagonal matrix with univariate GARCH processes on the diagonal. In other words, the distribution of $u_{t}$ conditional on past information has mean zero and covariance matrix

$$
\Sigma_{t \mid t-1}=B\left[\begin{array}{cc}
\Lambda_{t \mid t-1} & 0 \\
0 & I_{K-r}
\end{array}\right] B^{\prime}
$$

Thus, the reduced form error GARCH structure is driven by $r$ nontrivial GARCH components. This setup explicitly allows for the possibility of having fewer GARCH components than variables and, hence, accounts for the fact that some variables used in VAR analysis may be conditionally homoskedastic while others are conditionally heteroskedastic. 
To write down the GARCH components, we partition $B=\left[B_{1}: B_{2}\right]$ such that $B_{1}$ is $(K \times r)$ and $B_{2}$ is $(K \times(K-r))$ and let

$$
\mathbf{A}=\left[\begin{array}{l}
\mathbf{A}_{1} \\
\mathbf{A}_{2}
\end{array}\right]=B^{-1}
$$

be its inverse, partitioned such that $\mathbf{A}_{1}$ is $(r \times K)$ and $\mathbf{A}_{2}$ is $((K-r) \times K)$. Thus,

$$
\mathbf{A}_{1} u_{t}=\Lambda_{t \mid t-1}^{1 / 2} e_{1 t} \quad \text { and } \quad \mathbf{A}_{2} u_{t}=e_{2 t} .
$$

We assume that all the GARCH components are univariate $\operatorname{GARCH}(1,1)$ processes, that is,

$$
\sigma_{k, t \mid t-1}^{2}=\left(1-\gamma_{k}-g_{k}\right)+\gamma_{k}\left(\mathbf{a}_{k} u_{t-1}\right)^{2}+g_{k} \sigma_{k, t-1 \mid t-2}^{2}, \quad k=1, \ldots, r,
$$

where $\mathbf{a}_{k}$ is the $k$ th row of $\mathbf{A}_{1}$ and $\gamma_{k}>0, g_{k} \geq 0, \gamma_{k}+g_{k}<1$ so that the $\operatorname{GARCH}(1,1)$ processes are nontrivial for $k=1, \ldots, r$.

Sentana and Fiorentini (2001) and Milunovich and Yang (2013) show that if $r \geq K-1$, the matrix $B$ is unique up to permutations of the columns and column sign changes. Thus, the objective is to determine the number $r$ of GARCH components. If it turns out to be at least $K-1$ then the GARCH structure delivers fully identified structural shocks. Of course, in the present context, full identification means full local identification because the shocks are identified only up to sign changes and ordering. Therefore we use the terminology 'full identification' rather than the more conventional 'justidentification'. Notice that global identification can be achieved by fixing the sign of one element in each column of $B$ and by ordering the shocks in some unique way. The standardization of the signs means that the signs of shocks hitting the system are specified and the ordering has to be done by the analyst who has to decide where to position, for example, the monetary policy shock. Thus, this type of local identification is all we need for the present purposes. In the related literature sometimes the restrictions are imposed on $B^{-1}$. Often the uniqueness conditions include normalizing the main diagonal elements of this matrix to one (see, e.g., Milunovich and Yang (2013)).

Under our assumptions, the unconditional covariance matrix of $u_{t}$ is

$$
\Sigma_{u}=B B^{\prime}
$$

Following Lanne and Saikkonen (2007) we use a polar decomposition of $B=$ $C R$, where $C$ is a symmetric, positive definite $(K \times K)$ matrix and $R=\left[R_{1}\right.$ : $R_{2}$ ] is an orthogonal $(K \times K)$ matrix partitioned conformably with $B$ so that 
$B_{i}=C R_{i}, i=1,2$. Thus, $B B^{\prime}=C C$ so that $C=\Sigma_{u}^{1 / 2}$ is the unique square root matrix of $\Sigma_{u}$. The conditional covariance matrices can be written as

$$
\Sigma_{t \mid t-1}=\Sigma_{u}+C R_{1}\left(\Lambda_{t \mid t-1}-I_{r}\right) R_{1}^{\prime} C
$$

and

$$
u_{t}^{\prime} \Sigma_{t \mid t-1}^{-1} u_{t}=u_{t}^{\prime} \Sigma_{u}^{-1} u_{t}+u_{t}^{\prime} C^{-1} R_{1}\left(\Lambda_{t \mid t-1}^{-1}-I_{r}\right) R_{1}^{\prime} C^{-1} u_{t} .
$$

Thus, the Gaussian log-likelihood function of the model is

$$
\log L=\sum_{t=1}^{T} \log f_{t \mid t-1}\left(y_{t}\right)
$$

with

$$
\begin{aligned}
f_{t \mid t-1}\left(y_{t}\right)= & (2 \pi)^{-K / 2} \operatorname{det}\left(\Sigma_{t \mid t-1}\right)^{-1 / 2} \exp \left(-\frac{1}{2} u_{t}^{\prime} \Sigma_{t \mid t-1}^{-1} u_{t}\right) \\
= & (2 \pi)^{-K / 2} \operatorname{det}\left(\Sigma_{u}\right)^{-1 / 2} \exp \left(-\frac{1}{2} u_{t}^{\prime} \Sigma_{u}^{-1} u_{t}\right) \prod_{k=1}^{r} \sigma_{k, t \mid t-1}^{-1} \\
& \times \exp \left(-\frac{1}{2} u_{t}^{\prime} C^{-1} R_{1}\left(\Lambda_{t \mid t-1}^{-1}-I_{r}\right) R_{1}^{\prime} C^{-1} u_{t}\right) .
\end{aligned}
$$

Obviously, the log-likelihood depends on $\nu, \Pi_{1}, \ldots, \Pi_{p}, C, R_{1}$, and the GARCH parameters only, and not on $R_{2}$. This is important because, if there are just $r$ GARCH components, $R_{2}$ is not identified.

Estimation of high dimensional multivariate GARCH models by numerical optimization is time-consuming. Luckily the log-likelihood function factors into two parts: (i) a function of $C$, and (ii) a function of $C, R_{1}$ and $\Lambda_{t \mid t-1}$, such that one may break down the estimation in two main steps. First, $C$ is obtained as the unique square root of $\Sigma_{u}$, which is estimated as a sample covariance matrix. Second, conditional on the estimated $C$, the rows of $R_{1}$ and the GARCH equation parameters are estimated separately for $k=1, \ldots, r$. In fact, each equation $k+1$ is estimated conditionally on the previously estimated equation $k$. The exact procedure by which this is accomplished is described in Section 4 of Lanne and Saikkonen (2007). Although this approach is inefficent, it allows for relatively quick estimation of large systems and yields consistent estimates which may be used in the identification tests described next. 


\section{The Identification Tests}

Lanne and Saikkonen (2007) propose two different types of test statistics for testing hypotheses regarding the number of GARCH components $r$. Both test statistics can be viewed as LM type statistics because they require estimation only under $H_{0}: r=r_{0}$. The latter fact is important because otherwise potentially unidentified models have to be estimated. The parameters of a model with given $r$ are estimated by ML or some other, possibly less efficient procedure that provides estimators of $C$ and $R_{1}$ with $\sqrt{T}$ convergence rate. We denote the estimators by $\tilde{C}$ and $\tilde{R}_{1}$, respectively, and define

$$
\tilde{R}_{2}=\tilde{R}_{1 \perp}\left(\tilde{R}_{1 \perp}^{\prime} \tilde{R}_{1 \perp}\right)^{-1 / 2}
$$

where $\tilde{R}_{1 \perp}$ denotes an orthogonal complement of $\tilde{R}_{1}$, and

$$
\tilde{\mathbf{A}}_{2}=\tilde{R}_{2}^{\prime} \tilde{C}^{-1} .
$$

Recall that if $B$ is not identified, the same is true for $B^{-1}$. In that case $\tilde{\mathbf{A}}_{2}$ estimates some linear combination of the last $K-r$ rows of $B^{-1}$. However, if $\mathbf{A}_{2} u_{t}$ does not have GARCH, the same is true for any linear transformation. Hence, we estimate $\mathbf{A}_{2} u_{t}$ by $\tilde{\mathbf{A}}_{2} u_{t}$ and base a test of $H_{0}: r_{0}=r$ on this quantity because under this null hypothesis $\mathbf{A}_{2} u_{t}$ has no GARCH. In the following we continue to use the symbol $u_{t}$ for the residuals of the VAR model for simplicity. In practice they are, of course, replaced by the residuals from estimating the reduced-form $\operatorname{VAR}(p)$ in (1).

Following an idea of Ling and Li (1997), Lanne and Saikkonen (2007) propose a test statistic based on the autocovariances of quantities

$$
\xi_{t}=u_{t}^{\prime} \tilde{\mathbf{A}}_{2}^{\prime} \tilde{\mathbf{A}}_{2} u_{t}-T^{-1} \sum_{t=1}^{T} u_{t}^{\prime} \tilde{\mathbf{A}}_{2}^{\prime} \tilde{\mathbf{A}}_{2} u_{t} .
$$

More precisely, they consider the test statistic

$$
Q_{1}(H)=T \sum_{h=1}^{H}[\tilde{\gamma}(h) / \tilde{\gamma}(0)]^{2}
$$

and show that it is asymptotically $\chi^{2}(H)$ distributed if the null hypothesis is true. Here

$$
\tilde{\gamma}(h)=T^{-1} \sum_{t=h+1}^{T} \xi_{t} \xi_{t-h} .
$$


As mentioned before, the $u_{t}$ are replaced by estimated VAR residuals in practice. For our purposes a critical question is what are the actual small sample size and power properties when these tests are applied to macro data, for example.

Lanne and Saikkonen (2007) also propose another test based on the autocovariance matrices of the quantities

$$
\vartheta_{t}=\operatorname{vech}\left(\tilde{\mathbf{A}}_{2} u_{t} u_{t}^{\prime} \tilde{\mathbf{A}}_{2}^{\prime}\right)-T^{-1} \sum_{t=1}^{T} \operatorname{vech}\left(\tilde{\mathbf{A}}_{2} u_{t} u_{t}^{\prime} \tilde{\mathbf{A}}_{2}^{\prime}\right),
$$

where vech denotes the half-vectorization operator that stacks the elements of a symmetric $((K-r) \times(K-r))$ matrix from the diagonal downwards in a $\frac{1}{2}(K-r)(K-r+1)$-dimensional column vector. In this case, the test statistic has the form

$$
Q_{2}(H)=T \sum_{h=1}^{H} \operatorname{tr}\left[\tilde{\Gamma}(h)^{\prime} \tilde{\Gamma}(0)^{-1} \tilde{\Gamma}(h) \tilde{\Gamma}(0)^{-1}\right],
$$

where

$$
\tilde{\Gamma}(h)=T^{-1} \sum_{t=h+1}^{T} \vartheta_{t} \vartheta_{t-h}^{\prime} \quad \text { for } \quad h=0,1, \ldots,
$$

and $\tilde{\Gamma}(h)=\tilde{\Gamma}(-h)^{\prime}$ for $h<0$. Lanne and Saikkonen (2007) show that this statistic has an asymptotic

$$
\chi^{2}\left(\frac{1}{4} H(K-r)^{2}(K-r+1)^{2}\right)
$$

distribution under $H_{0}$.

A related test statistic is obtained by using the usual multivariate $\mathrm{ARCH}$ LM test based on the auxiliary model

$$
\eta_{t}=\delta_{0}+D_{1} \eta_{t-1}+\cdots+D_{H} \eta_{t-H}+\zeta_{t}
$$

where $\eta_{t}=\operatorname{vech}\left(\tilde{\mathbf{A}}_{2} u_{t} u_{t}^{\prime} \tilde{\mathbf{A}}_{2}^{\prime}\right), \delta_{0}$ is a $\frac{1}{2}(K-r)(K-r+1)$-dimensional fixed vector, the $D_{i}, i=1, \ldots, H$, are $\left(\frac{1}{2}(K-r)(K-r+1) \times \frac{1}{2}(K-r)(K-r+1)\right)$ parameter matrices and $\zeta_{t}$ is an error term. The standard LM statistic for testing the null hypothesis

$$
H_{0}: D_{1}=\cdots=D_{H}
$$

is

$$
L M(H)=\frac{1}{2} T(K-r)(K-r+1)-T \operatorname{tr}\left[\tilde{\Sigma}_{\zeta} \tilde{\Gamma}(0)^{-1}\right]
$$


where $\tilde{\Sigma}_{\zeta}$ is the estimated residual covariance matrix from model 7) (see Doornik and Hendry (1997)). Just like $Q_{2}(H)$, this test statistic is used with critical values from a

$$
\chi^{2}\left(\frac{1}{4} H(K-r)^{2}(K-r+1)^{2}\right)
$$

distribution. An $F$ version,

$$
F_{A R C H}(H)=L M(H) /\left[\frac{1}{4} H(K-r)^{2}(K-r+1)^{2}\right],
$$

to be used with critical values from an

$$
F\left(\frac{1}{4} H(K-r)^{2}(K-r+1)^{2}, T\right)
$$

distribution was also proposed (see Lütkepohl (2004)). For the present purposes SVAR-GARCH models can only be used when large sample sizes $T$ are available. For such sample sizes the differences between the $\chi^{2}$ and $F$ versions of the LM statistic should be minor. We have confirmed that with simulations related to those reported in the next section. Therefore we focus on the $\chi^{2}$ version in the following. Clearly, the large number of degrees of freedom in these tests make them most attractive for small $H$ and we will use $H=1$ exclusively. If there is higher order $\mathrm{ARCH}$, then there are typically also first order effects that can be captured by using $H=1$.

For our purposes tests of the null hypothesis $H_{0}: r=K-2$ are of particular interest because rejecting it and finding that the true rank is at least $K-1$ implies full identification of the structural shocks via the GARCH structure. Any conventional restrictions, for example, zero restrictions on the instantaneous effects matrix $B$, then become over-identifying even if they are just-identifying in a conventional framework. In the next section we explore the small sample properties of the alternative test statistics with a special focus on testing $H_{0}: r=K-2$.

\section{Monte Carlo Investigation of the Tests}

\subsection{Monte Carlo Design}

We assess the finite sample performance of the previously discussed tests by conducting Monte Carlo simulations of three- and five-dimensional processes, that is, $K=3$ or 5 . The objective is to see which one of the tests is best suited for investigating identification in SVAR models. Three sample sizes of $T=300,700$, and 1500 observations are used. For a limited set of Monte Carlo designs we also considered the sample size $T=5000$ to get a better 
insight into the convergence to the asymptotic properties of the tests. We do not report the results for $T=5000$ but refer to them where appropriate. Each experiment consists of 2000 replications.

All data generating processes (DGPs) are $\operatorname{VAR}(0)$ specifications, that is, we focus only on the innovations. They are generated as follows:

1. Independent random variables are drawn from a standard normal distribution and stacked into the vector $e_{t}=\left(e_{1, t}, \ldots, e_{K, t}\right)^{\prime}$.

2. GARCH processes are generated as

$$
\sigma_{k, t \mid t-1}^{2}=(1-\gamma-g)+\gamma \varepsilon_{k, t-1}^{2}+g \sigma_{k, t-1 \mid t-2}^{2},
$$

where $\varepsilon_{k, t}=e_{k, t} \sigma_{k, t \mid t-1}$ for $k=1, \ldots, K$. In other words, all GARCH components have the same parameter values in a particular design. Four sets of GARCH parameters are used:

$$
(\gamma, g)=(0.05,0.65),(0.10,0.70),(0.15,0.75),(0.17,0.80) .
$$

Notably the last two sets of parameter values result in rather persistent processes as they are occasionally observed in practice.

3. A sequence of $\Lambda_{r, t \mid t-1}^{1 / 2}$ is generated as $\Lambda_{r, t \mid t-1}^{1 / 2}=\operatorname{diag}\left(\sigma_{1, t \mid t-1}, \ldots, \sigma_{r, t \mid t-1}\right)$ for $r=1, \ldots, K$.

4. A sequence of data vectors $u_{t}^{(r)}=\left(u_{1, t}^{(r)}, \ldots, u_{K, t}^{(r)}\right)^{\prime}$ is computed according to equation (3) using each $\Lambda_{r, t \mid t-1}$ from Step 3 , and the $B$ matrix given by ${ }^{2}$

$$
B=\left[\begin{array}{rrrrr}
0.9 & 0.6 & -1.1 & 0.1 & -1.2 \\
-0.7 & 1 & 0.7 & 0.7 & 0.7 \\
-1.5 & -0.5 & 1.2 & -1.2 & -0.4 \\
0.4 & 0.5 & -0.2 & 0.5 & 0.4 \\
-0.5 & 1 & 0.5 & 0.9 & 1
\end{array}\right]
$$

for 5 -dimensional processes and its upper left-hand $(3 \times 3)$ submatrix for 3-dimensional processes.

For each data set $u_{t}^{(r)}, r=1, \ldots, K$, we compute the test statistics $Q_{1}(1)$, $Q_{2}(1)$, and $L M(1)$ as given in (5), (6), and (8), respectively. We follow Lanne and Saikkonen (2007) in setting $H=1$ when computing the test statistics. With this choice, even higher order ARCH effects can be picked up as long as they induce first order autocorrelation in the squared residuals.

\footnotetext{
${ }^{2}$ The elements of $B$ are chosen arbitrarily but such as to ensure a range of nonzero instantaneous effects of the shocks on the variables and invertibility of the matrix.
} 


\subsection{Size and Power for Three-dimensional Processes}

We consider the size and power of the tests by computing the rejection frequencies using the asymptotic $\chi^{2}$ distributions discussed in Section 3, and nominal sizes of $10 \%$ and $5 \%$. Table 1 reports the estimated test sizes for the three-dimensional processes, calculated as relative rejection frequencies of the true null hypothesis.

The results in Table 1 show that the rejection frequencies depend on the sample size, the GARCH parameters and the number $r$ of actual GARCH components in the process. In particular, we note the following:

- For testing $H_{0}: r=1$, the $Q_{1}(1)$ and $Q_{2}(1)$ tests tend to reject more often than specified by the nominal size. The tendency does not disappear in Table 1 with increasing sample size. Thus, even for $T=1500$ the tests tend to over-reject. We therefore explored the situation with even larger samples and a subset of DGPs and found that even for $T=5000$ there is a slight tendency to over-reject. The rejection frequencies do not increase with the sample size as can be conjectured from Table 1. However, the asymptotic $\chi^{2}$ distribution appears to be a good finite sample approximation for very large samples only.

- For $H_{0}: r=1, Q_{2}(1)$ tends to be slightly more liberal than $Q_{1}(1)$, that is, $Q_{2}(1)$ tends to reject more often than $Q_{1}(1)$. Since both tests have a tendency to over-reject in most cases, $Q_{1}(1)$ is somewhat preferable to the multivariate test version $Q_{2}(1)$ judging only by the test sizes.

- The $Q_{1}(1)$ and $Q_{2}(1)$ tests are identical except for rounding error if $K-r=1$. Thus, not surprisingly, the rejection frequencies for the two tests are almost identical for testing $H_{0}: r=2$.

- The rejection frequencies for the $L M(1)$ test tend to be lower than those of the $Q_{1}(1)$ and $Q_{2}(1)$ tests. As a consequence, $L M(1)$ is the most conservative test in situations where the rejection frequencies are below the nominal sizes and its tendency for over-rejection is smaller in cases where the tests reject too often.

- The rejection frequencies depend on the GARCH parameters but are not monotone with respect to the persistence (measured by $\gamma+g$ ) of the processes. For example, looking at the results for a nominal size of $10 \%$ and a sample size of $T=700$, all tests reject most often for the intermediate parameters $(\gamma, g)=(0.10,0.70)$.

Summarizing the results for the test sizes, all three tests do not distort the size dramatically for large samples. Since the multivariate test version $Q_{2}(1)$ 
tends to over-reject more than $Q_{1}(1)$ the latter test is slightly preferable to the former. A good balance between over- and under-rejection is achieved with the $L M(1)$ test.

Of course, the size of a test is just one criterion. Therefore we present power results in Table 2. With respect to the issue of identification the question of primary importance is if there are more than $K-2 \mathrm{GARCH}$ components. in that case the structural shocks are fully identified via the volatility structure. Therefore we present results for the null hypothesis $H_{0}: r=1(=K-2)$ in Table 2, Rejecting the null hypothesis implies that full identification is found. The following results can be deduced from Table 2 ,

- Not surprisingly, those tests that over-reject (that is, those tests that actually have a larger size than the nominal one) also tend to reject more often when the null hypothesis is false and, hence, they have seemingly more power. In particular, $Q_{2}(1)$ rejects more often than $Q_{1}(1)$ which in turn tends to reject more than $L M(1)$.

- The power of all three tests tends to increase with the persistence $(\gamma+g)$ and is rather low for processes with low persistence (small $\gamma+g)$. This can be seen in Table 2 for GARCH parameters $(\gamma, g)=(0.05,0.65)$ and $(\gamma, g)=(0.10,0.70)$. For the first set of parameters the power is even low for the largest sample size $T=1500$. Thus, using these tests it is quite possible to miss a GARCH component that has low persistence. In practice, however, quite persistent processes are often observed which is good news for the power of the tests.

- The power increases with the sample size and the number of GARCH components for all three tests. $Q_{2}(1)$ retains higher rejection frequencies than $Q_{1}(1)$ even in the samples of $T=1500$ observations for most null hypotheses.

Thus, overall for three-dimensional processes there is not much to choose between the tests for our purposes. In terms of meeting the nominal size, $Q_{1}(1)$ and $L M(1)$ have advantages whereas $Q_{2}(1)$ rejects more often when the alternative is true. The latter result has to be qualified, however, because we are not considering size-adjusted rejection frequencies and $Q_{2}(1)$ often overrejects under $H_{0}$. Of course, the small sample properties of the tests may well depend on the dimension of the process as well. Therefore we present results for five-dimensional processes next. 


\subsection{Size and Power for Five-dimensional Processes}

Relative rejection frequencies of the tests for five-dimensional processes are presented in Tables 3 and 4 . Because the critical question for full identification of the shocks for a five-dimensional process is whether there are at least four GARCH components, we focus on tests of the null hypothesis $H_{0}: r=3$. If that hypothesis is rejected and the true number of GARCH components $r>3$, then we have full identification. Thus, to avoid sequential testing, one may be inclined to focus only on this null hypothesis in the present context. In Tables 3 and 4 relative rejection frequencies are presented for true numbers of GARCH components of $r=1, \ldots, 5$. Thus, the tables give an impression of test sizes in small samples for $r=3$ and of test power for $r=4$ and 5 . For $r=1,2$ we now look at situations where the true number of GARCH components is smaller than the one specified under $H_{0}$.

In Table 3 we present results for processes with lower persistence $[(\gamma, g)=$ $(0.05,0.65)$ and $(0.10,0.70)]$ while rejection frequencies for more persistent processes $[(\gamma, g)=(0.15,0.75)$ and $(0.17,0.80)]$ are given in Table 4 . The following observations emerge from the tables:

- In general $L M(1)$ has lower rejection frequencies than $Q_{1}(1)$ which in turn tends to reject less frequently than $Q_{2}(1)$. In particular, for small samples of $T=300$ this ordering is not always valid, however. For situations where the true $r$ is lower than specified in $H_{0}(r=1$ or 2 ) the $L M(1)$ test tends to reject less frequently than the corresponding nominal sizes indicate. Thus, $Q_{1}(1)$ and $Q_{2}(1)$ come closer to the nominal sizes for these cases. On the other hand they tend to overreject when the null hypothesis is true, that is, $r=3$. They also reject more frequently for $r>3$ and thus have power greater than $L M(1)$ in most cases. Note, however, that this is not size-adjusted power and is hence not surprising given the higher rejection frequencies for true null hypotheses.

- Comparing only $Q_{1}(1)$ and $Q_{2}(1)$ it appears again that the former has some advantages in terms of keeping the size. One may in fact prefer $Q_{1}(1)$ over $Q_{2}(1)$ because of its better size properties. This result is analogous to the three-dimensional case.

- The low power for GARCH processes with lower persistence in Table 3 is quite striking. In particular, for $(\gamma, g)=(0.05,0.65)$ in Table 3, the power remains low even for larger samples of size $T=1500$. The chances to find the GARCH components are low if they are not very persistent. For more persistent processes the problem is alleviated, as can be seen in Table 4 . 


\subsection{Estimating the Number of GARCH Components}

So far we have focussed on testing for full identification. If the tests do not support full identification, then one may want to explore the number of GARCH components and test sequentially larger numbers $r$ starting with $H_{0}: r=1$. The procedure stops when a given null hypothesis cannot be rejected. Thereby we get an estimate of the number of GARCH components. Even if the number is smaller than $K-1$, that can be useful information because even a smaller number of GARCH components provides some identifying information. We will now explore the ability of the tests to estimate the true number of GARCH components in such a sequential procedure.

We use a constant significance level of $5 \%$ for all tests. ${ }^{3}$ Tables 5 and 6 display relative frequencies of the numbers of GARCH components estimated in this way for five-dimensional processes with GARCH parameters $(\gamma, g)=$ $(0.1,0.7)$ and $(\gamma, g)=(0.17,0.80)$, respectively, and three sample sizes, $T=$ $300,700,1500$. Thus, in Table 5 we consider processes for which the power of the tests is relatively low whereas it is a bit higher for the processes in Table 6.

Considering the diagonal entries across all panels of Table 5, we observe that overall $Q_{2}(1)$ outperforms $Q_{1}(1)$ and $L M(1)$ in terms of estimating the true number of GARCH components correctly. In fact, sometimes $Q_{2}(1)$ chooses the correct number of GARCH components more than twice as often as $Q_{1}(1)$ and it is also considerably superior to $L M(1)$. The latter criterion clearly outperforms $Q_{1}(1)$. It cannot be overlooked however, that all three criteria are doing poorly in finding the right model if the sample size is small $(T=300)$ or even moderate $(T=700)$. Even for the largest sample size $(T=1500)$ they are not very reliable in finding the correct number of GARCH components if that number is greater than 1. For sample sizes $T=700$ and 1500 , there appears to be a U-shaped pattern in the frequencies across diagonal entries, with the best outcomes reached for $r=1$ and 5 GARCH components, and the worst for $r=2,3$ or 4 .

All numbers below the diagonals in the panels of Table 5 are remarkably close to zero. Thus, all three testing procedures rarely over-estimate the true number of GARCH components. In contrast, under-estimating $r$ is quite common, as can be seen by looking at the above-diagonal elements in all panels of Table 5 .

If the persistence of the GARCH components increases, the ability of the three testing procedures to locate the correct model increases substantially,

3 A sequential procedure such as the one used here estimates the true number of GARCH components consistently provided the significance level $\alpha_{T}$ is adjusted with increasing sample size such that $\lim _{T \rightarrow \infty} \frac{\log \alpha_{T}}{T}=0$, as discussed in Hosoya (1989). 
as can be seen in Table 6. Even there the performance of the procedures is far from satisfactory for samples as small as $T=300$ observations. For example, for $T=300, Q_{1}(1), Q_{2}(1)$, and $L M(1)$ find a correct number of $4 \mathrm{GARCH}$ components in $11.4 \%, 25.0 \%$, and $14.4 \%$, respectively, of the replications of our simulation experiment. The corresponding numbers for $T=1500$ are $93.0 \%, 94.1 \%$, and $93.6 \%$, respectively, and, thus, are substantially better. Notice, however, that even in Table 6 the above-diagonal elements of the panels tend to be markedly larger than the below-diagonal elements for samples smaller than $T=1500$. Hence, under-estimation of the true number of GARCH components is more common than over-estimation of $r$.

Overall these results reflect the poor power of the tests for processes with low persistence in the GARCH structure. Also, the preferable performance of $Q_{2}(1)$ is driven by its tendency to over-reject. Hence, it is effectively working with a different significance level and, thus, the larger number of rejections, which in turn imply larger estimated values of $r$, is no surprise. In fact, the results indicate that using a larger significance level for the tests may result in larger probabilities to find the correct number of GARCH components.

We have also performed simulations with processes with even lower persistence $[(\gamma, g)=(0.05,0.65)]$ and with $(\gamma, g)=(0.15,0.75)$ to confirm that the results are in line with those for the processes shown in Tables 5 and 6 . As expected, the performance of the testing procedures is worst for the process with least persistence and they have the highest probability of finding the correct number of GARCH components for the most persistent process. Therefore we do not present tables with detailed results.

Thus, the overall picture evolving from the simulations based on fivedimensional DGPs is similar to the three-dimensional case. In other words, $L M(1)$ has lower rejection frequencies than $Q_{1}(1)$ which in turn rejects less frequently than $Q_{2}(1)$. Since the tests tend to over-reject, this gives a slight advantage to $L M(1)$ in terms of precision in meeting the nominal test size. In turn, $L M(1)$ is somewhat conservative in many situations and therefore its power is also somewhat lower than that of the other two tests. The most liberal test based on $Q_{2}(1)$ has the best chances of finding the correct number of GARCH components in the sequential testing procedure. Since none of the three tests is uniformly superior, it is perhaps best to perform different tests and keep their small sample properties in mind when interpreting the results. Given the generally low power of the tests it can be concluded that rejecting under-identified models is strong evidence against them and in favour of full identification if that is the alternative. 


\section{An Application}

We apply the above discussed tests to investigate identification of the SVARGARCH models used in a recently published paper by Bouakez and Normandin (2010) who examine the importance of U.S. monetary policy shocks for exchange rate dynamics. We use this example as an illustration of our tests and their implications for structural analysis. We are fully aware that the example system has features that are not covered by the previous analysis. For example, the VAR processes for our example systems may have unit roots. We ignore such additional complications and focus on the structural identification issues. Clearly, Bouakez and Normandin (2010) perform a much richer economic analysis that we do not question with our investigation.

The baseline model is 8-dimensional. It includes variables of a standard U.S. monetary system for identifying the monetary policy shocks and variables representing the foreign exchange market of a number of countries. More precisely, they use the following variables for the U.S. and G7 countries:

$q_{t}-\log$ of U.S. industrial production index,

$p_{t}-\log$ of U.S. consumer price index,

$c p_{t}-\log$ of world export commodity price index,

$n b r_{t}-\log$ of U.S. nonborrowed reserves,

$t r_{t}-\log$ of U.S. total reserves,

$f f_{t}$ - federal funds rate,

$d r_{t}$ - difference between foreign short-term interest rate and U.S. threemonths Treasury Bill rate,

$e x_{t}-\log$ of exchange rate (U.S. dollars per one unit of foreign currency).

Conventional identification restrictions are often imposed on $B^{-1}$ in the setting $B^{-1} u_{t}=\varepsilon_{t}$, where $u_{t}=\left(u_{q, t}, u_{p, t}, u_{c p, t}, u_{n b r, t}, u_{t r, t}, u_{f f, t}, u_{d r, t}, u_{e x, t}\right)^{\prime}$ and $\varepsilon_{t}$ is an 8-dimensional vector of structural shocks. Different sets of conventional identifying restrictions on $B^{-1}$ are reviewed by Bouakez and Normandin (2010). They summarize them in three groups:

Equilibrium restrictions: Based on Bernanke and Mihov (1998) the model for the market for bank reserves being in equilibrium implies restrictions on $B^{-1}$. 
Targeting restrictions: Depending on which monetary targeting indicator is used, competing sets of restrictions are imposed to specify the targeting variable of the monetary authority.

Orthogonality restrictions: The policy variables $n b r_{t}, t r_{t}, f f_{t}, d r_{t}, e x_{t}$ are restricted to have no contemporaneous effect on the goods variables $q_{t}, p_{t}, c p_{t}$. Moreover, the Fed is assumed to respond with a delay to changes in the interest rate differential and exchange rates. Hence, the instantaneous effects of $d r_{t}$ and $e x_{t}$ on $n b r_{t}, t r_{t}$, and $f f_{t}$ are restricted to zero.

Bouakez and Normandin (2010) use the GARCH setup to investigate the validity of these sets of conventional restrictions. Specifically, they consider monthly observations for periods 1982:11 - 1998:12 for the euro area countries France, Italy, and Germany and for 1982:11 - 2004:10 for the remaining countries, Canada, Japan, and UK. They fit VAR(6) models with seven $\operatorname{GARCH}(1,1)$ components and argue that their models fully identify the shocks.

Their argument in favour of having a fully identified model relies on a criterion given by Sentana and Fiorentini (2001). These authors define the $(T \times K)$ matrix $\Gamma$ such that its $k$ th column consists of the conditional variances of the $k$ th structural shock, $\left(\sigma_{k, 1 \mid 0}^{2}, \ldots, \sigma_{k, T \mid T-1}^{2}\right)^{\prime}$, and show that full identification is equivalent to $\Gamma^{\prime} \Gamma$ having full rank. Bouakez and Normandin (2010) do not check the rank of the actual matrix $\Gamma^{\prime} \Gamma$ but replace the true conditional variances by estimated quantities. They find that the estimated matrix has full rank. However, a formal statistical test of the rank condition would have to test the rank of the true underlying $\Gamma$ matrix so that their rank check is not a formal statistical test. In fact, estimating the elements of a reduced-rank matrix unrestrictedly typically results in a full rank matrix even if the true matrix of interest has reduced rank, see e.g. Cragg and Donald (1997). Thus, a formal statistical test is called for. It can be performed as discussed in the previous sections and we will return to that shortly.

Before we perform formal identification tests it may be worth pointing out some conclusions drawn by Bouakez and Normandin (2010). Under the assumption of fully identified shocks via the GARCH structure the equilibrium, targeting, and orthogonality restrictions are over-identifying, of course, and, hence, can be tested against the data. They find that the equilibrium restrictions cannot be rejected for any of the six G7 countries considered while the targeting and orthogonality restrictions are rejected for all countries.

Because these results rest on the assumption of full identification via the GARCH structure we will now apply our tests to explore this condition. Notice that for some but not for all of the variables included in the models 
GARCH errors are quite plausible. In particular, GARCH is often found in financial market variables observed at monthly frequency. On the other hand, one would not necessarily expect GARCH in variables such as industrial production $\left(q_{t}\right)$ or the consumer price index $\left(p_{t}\right)$. In fact, many of the estimated GARCH parameters in Table 1 of Bouakez and Normandin (2010) are not significant in the sense that they are smaller than twice their standard errors. Thus, one may wonder about the validity of the identification conditions for the shocks.

We use the same data and models and apply our three tests for the number of GARCH components.4 Estimation is done by the Gaussian ML procedure discussed in Section 2.2 in Ox 7.0, using the MaxSQP function, which implements a sequential quadratic programming technique to maximize a non-linear function subject to non-linear constraints. The non-linear constraints are introduced by the sequential estimation of the columns of $R_{1}$ and the GARCH parameters. Prior to the maximization of the log-likelihood function, a VAR(6) model is fitted by OLS for each country and the residuals are then used in the subsequent analysis. The resulting $p$-values are presented in Table 7 together with the estimated persistences of the GARCH components. Recall that for full identification we have to have more than 6 GARCH components. Hence we should reject $H_{0}: r=6$. Looking at the corresponding $p$-values based on the asymptotic $\chi^{2}$ distributions in Table 7 it is clear that all tests for all countries except for Japan result in $p$-values that do not justify rejection of the null hypothesis at conventional significance levels. In particular, all $p$-values are greater than $10 \%$. The single exception is the model for Japan for which $Q_{1}(1)$ has a $p$-value of 0.047 and also the $p$-values of $L M(1)$ and $Q_{2}(1)$ are around 5\%. Thus, only for Japan more than six GARCH components can be justified on the basis of our tests. Taking into account the low power of the tests, the result for Japan can in fact be seen as strong evidence in favour of full identification. Of course, the low power of the tests may also be used as an argument in favor of larger significance levels. Indeed the $p$-value of a $Q_{1}(1)$ test of $H_{0}: r=6$ for Germany is just a little larger than $10 \%$. Hence, one could also argue that there is evidence of full identification for Germany.

In Table 7 we present $p$-values for null hypotheses ranging from $r=1$ to $r=7$ and it turns out that only rather small numbers of GARCH components can be justified by our test results for most countries. For example, $H_{0}: r=4$ is not rejected for Canada while $H_{0}: r=3$ is not rejected for France and Italy at conventional significance levels. Considering the properties of the tests it may also be worth pointing out that some of the estimated

\footnotetext{
${ }^{4}$ We thank Hafedh Bouakez for providing the data.
} 
GARCH components in the models underlying the test results are highly persistent with estimated $\gamma+g>0.9$. The estimated persistence of the $r$ th GARCH process for each of the models is also reported in Table 7 . Since the estimated GARCH components are not updated when $r$ increases, the persistence of all the estimated GARCH components is in fact seen in Table 7. For example, the two GARCH components for France for $r=2$ have persistence $\gamma_{1}+g_{1}=0.986$ and $\gamma_{2}+g_{2}=0.990$. Given the size of these persistence estimates and based on our simulation results one would expect to have a chance to find more GARCH components if they are actually driving the volatility in the DGPs. On the other hand, the sample sizes are even smaller than in the Monte Carlo simulations and we know from the simulations that the tests may not have much power for small samples. Despite this limitation of the tests they clearly cannot be used to support the assumption of full identification via the GARCH structure for most of the countries, the exceptions being Japan and perhaps Germany.5

Therefore it may be worth discussing the implications of not having full identification. First, given that there are some GARCH components in all of the country models, there is some identifying information from the GARCH structure. Hence it is not surprising that some of the conventional restrictions are rejected by Bouakez and Normandin (2010). In other words, even without full identification there is enough curvature in the likelihood to reject the targeting and orthogonality restrictions. These rejections are clear evidence against the targeting and orthogonality restrictions even without a fully identified model. On the other hand, not rejecting the equilibrium restrictions may just be a consequence of lack of identification and, hence, lack of sufficient curvature in the likelihood.

Since the equilibrium restrictions were not rejected, Bouakez and Normandin (2010) use them in an impulse response analysis based on a $B$ matrix which is otherwise identified by the GARCH structure. Even with the equilibrium restrictions imposed the shocks will not be unique without additional restrictions from the GARCH structure, of course. Given that the GARCH structure is not economically motivated, it is not clear that the shocks ob-

\footnotetext{
${ }^{5}$ Since our tests have little power in small samples, we have extended the samples for Canada, Japan, and the UK to May 2013. Also for the extended samples seven GARCH components are not supported for Canada and the UK while for Japan the evidence in favour of more than six GARCH components vanishes. More precisely, the $p$-values for $H_{0}: r=6$ increase above $50 \%$ for Japan. This may be partly due to the fact that the extended data series are not identical to those used in the original study by Bouakez and Normandin (2010). Therefore we do not report detailed results. For France, Germany, and Italy an extension of the sample size is not possible because the currencies ceased to exist in 1999 when the euro replaced them.
} 
tained in this way correspond to meaningful economic shocks. Under the assumptions of Bouakez and Normandin (2010), the GARCH structure identifies not only one shock and the problem arises which one of the shocks deserves the label 'monetary policy shock'. They make the choice on the basis of assumptions on the Fed's feedback rule and find that the responses to monetary policy shocks obtained in their setting are quite different from what is reported in other studies based on conventional identifying restrictions.

If, however, their assumption of having fully identified shocks via the GARCH structure is incorrect, then not rejecting the equilibrium restrictions may just be a consequence of lack of identification and imposing the restrictions may be as problematic as in a conventional setting. Moreover, without fully identified shocks via GARCH, it is not clear whether the impulse responses depicted in Figures 2 and 3 of Bouakez and Normandin (2010) reflect realistically what is going on in the economy and in particular in the exchange market. Thus, their conclusion that an expansionary monetary policy shock leads to a 'delayed overshooting of the nominal exchange rate, with a peak occurring at around 10 months after the shock and to large deviations from UIP' [uncovered interest rate parity] (p. 152) is on firm grounds only for the Japanese and perhaps the German system. For all other systems the situation is much less clear in the light of the problematic identification assumptions. Even the Japanese and German cases rest on the assumption that none of the other shocks qualifies as a monetary policy shock which cannot be concluded from the identifying information obtained from the GARCH structure alone.

\section{Conclusions}

This study contributes to the growing literature on identification of structural shocks in SVAR analysis via changes in volatility. We consider GARCH models for the changes in volatility and argue that formal statistical tests for identification are important in this context while informal criteria that have been used in the past are in general insufficient. We point out that tests proposed by Lanne and Saikkonen (2007) are suitable for testing for identification in the present context and we explore their small sample properties and compare them to another test we consider as a plausible alternative. We find that the small sample properties of all three tests compared in our simulation study depend on the sample size, the persistence properties and structure of the underlying GARCH process. In small samples they may all have relatively little power and tend to favour under-identified models over fully identified models. Given their dependence on unknown properties of the 
DGP and the fact that none of them is uniformly dominating its competitors, it may be a good strategy to apply all of them. Since they all have low power, if they reject an under-identified model in favour of a fully identified model this is strong evidence of having full identification in samples of the size typically available in macroeconometric studies.

We have reconsidered a study investigating the effects of U.S. monetary policy on exchange rates for which the SVAR-GARCH approach to identification of structural shocks has been used. We argue that variables such as industrial production and the consumer price index may not have independent GARCH components. Hence, using them in a VAR system may result in GARCH residuals that are driven by a smaller number of GARCH components. This is precisely the situation where fully identified shocks are not obtained from the GARCH structure. We apply our formal statistical tests for identification. Depending on the significance level used, we confirm full identification only for one or two out of six countries. To demonstrate how the approach can be used in such a case we discuss in detail which of the conclusions of the previous study can be maintained and which ones are problematic. In particular, it is argued that the previous conclusions regarding the responses of the exchange rates to a monetary policy shock are problematic if the shocks are not fully identified.

\section{References}

Bernanke, B. S. and Mihov, I. (1998). Measuring monetary policy, Quarterly Journal of Economics 113: 869-902.

Bouakez, H. and Normandin, M. (2010). Fluctuations in the foreign exchange market: How important are monetary policy shocks?, Journal of International Economics 81: 139-153.

Cragg, J. G. and Donald, S. G. (1997). Inferring the rank of a matrix, Journal of Econometrics 76: 223-250.

Doornik, J. A. and Hendry, D. F. (1997). Modelling Dynamic Systems Using PcFiml 9.0 for Windows, International Thomson Business Press, London.

Hosoya, Y. (1989). Hierarchical statistical models and a generalized likelihood ratio test, Journal of the Royal Statistical Society B 51: 435 447. 
Lanne, M. and Lütkepohl, H. (2008). Identifying monetary policy shocks via changes in volatility, Journal of Money, Credit and Banking 40: 11311149 .

Lanne, M., Lütkepohl, H. and Maciejowska, K. (2010). Structural vector autoregressions with Markov switching, Journal of Economic Dynamics and Control 34: 121-131.

Lanne, M. and Saikkonen, P. (2007). A multivariate generalized orthogonal factor GARCH model, Journal of Business 83 Economic Statistics 25: $61-75$.

Ling, S. and Li, W. K. (1997). Diagnostic checking of nonlinear multivariate time series with multivariate ARCH errors, Journal of Time Series Analysis 18: 447-464.

Lütkepohl, H. (2004). Vector autoregressive and vector error correction models, in H. Lütkepohl and M. Krätzig (eds), Applied Time Series Econometrics, Cambridge University Press, Cambridge, pp. 86-158.

Milunovich, G. and Yang, M. (2013). On identifying structural VAR models via ARCH effects, Journal of Time Series Econometrics 5: 117-131.

Normandin, M. and Phaneuf, L. (2004). Monetary policy shocks: Testing identification conditions under time-varying conditional volatility, Journal of Monetary Economics 51: 1217-1243.

Rigobon, R. (2003). Identification through heteroskedasticity, Review of Economics and Statistics 85: 777-792.

Sentana, E. and Fiorentini, G. (2001). Identification, estimation and testing of conditionally heteroskedastic factor models, Journal of Econometrics 102: $143-164$.

Sims, C. A. (1980). Macroeconomics and reality, Econometrica 48: 1-48. 
Table 1: Estimated Sizes of the Tests for Three-Dimensional Processes

\begin{tabular}{|c|c|c|c|c|c|c|c|c|}
\hline \multirow{3}{*}{$\begin{array}{l}\text { GARCH } \\
\text { parameters }\end{array}$} & \multirow[b]{3}{*}{$H_{0}$} & \multirow[b]{3}{*}{$T$} & \multicolumn{6}{|c|}{ Nominal Size } \\
\hline & & & \multicolumn{3}{|c|}{$10 \%$} & \multicolumn{3}{|c|}{$5 \%$} \\
\hline & & & $L M(1)$ & $\overline{Q_{1}(1)}$ & $Q_{2}(1)$ & $L M(1)$ & $\overline{Q_{1}(1)}$ & $Q_{2}(1)$ \\
\hline \multirow{6}{*}{$\begin{array}{l}\gamma=0.05 \\
g=0.65\end{array}$} & $r=1$ & 300 & 0.073 & 0.100 & 0.110 & 0.044 & 0.054 & 0.063 \\
\hline & & 700 & 0.083 & 0.108 & 0.119 & 0.052 & 0.051 & 0.068 \\
\hline & & 1500 & 0.110 & 0.124 & 0.149 & 0.062 & 0.066 & 0.097 \\
\hline & $r=2$ & 300 & 0.073 & 0.089 & 0.089 & 0.034 & 0.045 & 0.046 \\
\hline & & 700 & 0.095 & 0.119 & 0.119 & 0.049 & 0.063 & 0.063 \\
\hline & & 1500 & 0.101 & 0.133 & 0.133 & 0.058 & 0.072 & 0.072 \\
\hline \multirow{6}{*}{$\begin{array}{l}\gamma=0.10 \\
g=0.70\end{array}$} & $r=1$ & 300 & 0.084 & 0.115 & 0.131 & 0.054 & 0.062 & 0.080 \\
\hline & & 700 & 0.104 & 0.125 & 0.139 & 0.072 & 0.068 & 0.097 \\
\hline & & 1500 & 0.111 & 0.141 & 0.157 & 0.081 & 0.082 & 0.108 \\
\hline & $r=2$ & 300 & 0.083 & 0.096 & 0.096 & 0.048 & 0.060 & 0.060 \\
\hline & & 700 & 0.099 & 0.127 & 0.127 & 0.066 & 0.079 & 0.079 \\
\hline & & 1500 & 0.112 & 0.138 & 0.138 & 0.077 & 0.087 & 0.087 \\
\hline \multirow{6}{*}{$\begin{aligned} \gamma & =0.15 \\
g & =0.75\end{aligned}$} & $r=1$ & 300 & 0.087 & 0.111 & 0.125 & 0.056 & 0.061 & 0.076 \\
\hline & & 700 & 0.092 & 0.118 & 0.124 & 0.063 & 0.062 & 0.081 \\
\hline & & 1500 & 0.083 & 0.122 & 0.119 & 0.049 & 0.059 & 0.070 \\
\hline & $r=2$ & 300 & 0.085 & 0.108 & 0.109 & 0.053 & 0.064 & 0.064 \\
\hline & & 700 & 0.080 & 0.107 & 0.107 & 0.050 & 0.061 & 0.062 \\
\hline & & 1500 & 0.082 & 0.103 & 0.106 & 0.046 & 0.055 & 0.055 \\
\hline \multirow{6}{*}{$\begin{array}{l}\gamma=0.17 \\
g=0.80\end{array}$} & $r=1$ & 300 & 0.081 & 0.116 & 0.121 & 0.055 & 0.058 & 0.075 \\
\hline & & 700 & 0.081 & 0.120 & 0.113 & 0.052 & 0.061 & 0.071 \\
\hline & & 1500 & 0.073 & 0.108 & 0.110 & 0.041 & 0.053 & 0.060 \\
\hline & $r=2$ & 300 & 0.092 & 0.110 & 0.111 & 0.050 & 0.061 & 0.061 \\
\hline & & 700 & 0.072 & 0.094 & 0.095 & 0.045 & 0.054 & 0.054 \\
\hline & & 1500 & 0.079 & 0.098 & 0.097 & 0.041 & 0.051 & 0.051 \\
\hline
\end{tabular}


Table 2: Estimated Powers of the Tests for Null Hypothesis $H_{0}: r=1$ Based on Three-Dimensional Processes

\begin{tabular}{|c|c|c|c|c|c|c|c|c|}
\hline \multirow{3}{*}{$\begin{array}{l}\text { GARCH } \\
\text { parameters }\end{array}$} & \multirow[b]{3}{*}{ true $r$} & \multirow[b]{3}{*}{$T$} & \multicolumn{6}{|c|}{ Nominal Size } \\
\hline & & & \multicolumn{3}{|c|}{$10 \%$} & \multicolumn{3}{|c|}{$5 \%$} \\
\hline & & & $L M(1)$ & $\overline{Q_{1}(1)}$ & $\overline{Q_{2}(1)}$ & $L M(1)$ & $Q_{1}(1)$ & $\overline{Q_{2}(1)}$ \\
\hline$\gamma=0.05$ & 2 & 300 & 0.098 & 0.126 & 0.135 & 0.063 & 0.069 & 0.089 \\
\hline \multirow[t]{5}{*}{$g=0.65$} & & 700 & 0.141 & 0.161 & 0.195 & 0.088 & 0.093 & 0.127 \\
\hline & & 1500 & 0.230 & 0.225 & 0.303 & 0.157 & 0.149 & 0.211 \\
\hline & 3 & 300 & 0.124 & 0.162 & 0.170 & 0.083 & 0.099 & 0.112 \\
\hline & & 700 & 0.229 & 0.275 & 0.293 & 0.164 & 0.191 & 0.211 \\
\hline & & 1500 & 0.421 & 0.418 & 0.509 & 0.319 & 0.387 & 0.390 \\
\hline \multirow{6}{*}{$\begin{array}{l}\gamma=0.10 \\
g=0.70\end{array}$} & 2 & 300 & 0.200 & 0.182 & 0.258 & 0.142 & 0.109 & 0.184 \\
\hline & & 700 & 0.406 & 0.345 & 0.478 & 0.325 & 0.254 & 0.384 \\
\hline & & 1500 & 0.745 & 0.619 & 0.815 & 0.683 & 0.515 & 0.755 \\
\hline & 3 & 300 & 0.334 & 0.384 & 0.410 & 0.261 & 0.280 & 0.316 \\
\hline & & 700 & 0.679 & 0.736 & 0.772 & 0.601 & 0.644 & 0.686 \\
\hline & & 1500 & 0.949 & 0.964 & 0.981 & 0.922 & 0.940 & 0.959 \\
\hline \multirow{6}{*}{$\begin{array}{l}\gamma=0.15 \\
g=0.75\end{array}$} & 2 & 300 & 0.410 & 0.387 & 0.494 & 0.334 & 0.297 & 0.404 \\
\hline & & 700 & 0.776 & 0.700 & 0.843 & 0.718 & 0.613 & 0.784 \\
\hline & & 1500 & 0.974 & 0.956 & 0.992 & 0.963 & 0.924 & 0.987 \\
\hline & 3 & 300 & 0.674 & 0.703 & 0.764 & 0.602 & 0.625 & 0.693 \\
\hline & & 700 & 0.962 & 0.981 & 0.992 & 0.950 & 0.967 & 0.984 \\
\hline & & 1500 & 0.996 & 1.000 & 1.000 & 0.996 & 1.000 & 1.000 \\
\hline \multirow{6}{*}{$\begin{array}{l}\gamma=0.17 \\
g=0.80\end{array}$} & 2 & 300 & 0.557 & 0.533 & 0.638 & 0.492 & 0.448 & 0.571 \\
\hline & & 700 & 0.920 & 0.892 & 0.958 & 0.898 & 0.853 & 0.937 \\
\hline & & 1500 & 0.994 & 0.997 & 0.999 & 0.994 & 0.993 & 0.999 \\
\hline & 3 & 300 & 0.825 & 0.848 & 0.895 & 0.787 & 0.791 & 0.856 \\
\hline & & 700 & 0.984 & 0.999 & 1.000 & 0.983 & 0.994 & 0.999 \\
\hline & & 1500 & 0.995 & 1.000 & 1.000 & 0.995 & 1.000 & 1.000 \\
\hline
\end{tabular}


Table 3: Relative Rejection Frequencies of the Tests for Null Hypothesis $H_{0}: r=3$ Based on Five-Dimensional Processes with Low Persistence

\begin{tabular}{|c|c|c|c|c|c|c|c|c|}
\hline \multirow{3}{*}{$\begin{array}{l}\text { GARCH } \\
\text { parameters }\end{array}$} & \multirow[b]{3}{*}{ true $r$} & \multirow[b]{3}{*}{$T$} & \multicolumn{6}{|c|}{ Nominal Size } \\
\hline & & & \multicolumn{3}{|c|}{$10 \%$} & \multicolumn{3}{|c|}{$5 \%$} \\
\hline & & & $L M(1)$ & $Q_{1}(1)$ & $\overline{Q_{2}(1)}$ & $L M(1)$ & $Q_{1}(1)$ & $Q_{2}(1)$ \\
\hline \multirow{15}{*}{$\begin{array}{l}\gamma=0.05 \\
g=0.65\end{array}$} & \multirow[t]{3}{*}{1} & 300 & 0.053 & 0.108 & 0.080 & 0.030 & 0.052 & 0.042 \\
\hline & & 700 & 0.060 & 0.095 & 0.082 & 0.035 & 0.050 & 0.047 \\
\hline & & 1500 & 0.084 & 0.080 & 0.086 & 0.029 & 0.043 & 0.044 \\
\hline & \multirow[t]{3}{*}{2} & 300 & 0.054 & 0.092 & 0.079 & 0.026 & 0.045 & 0.040 \\
\hline & & 700 & 0.058 & 0.093 & 0.086 & 0.030 & 0.043 & 0.044 \\
\hline & & 1500 & 0.077 & 0.111 & 0.112 & 0.042 & 0.063 & 0.065 \\
\hline & \multirow[t]{3}{*}{3 (size) } & 300 & 0.056 & 0.092 & 0.087 & 0.033 & 0.043 & 0.046 \\
\hline & & 700 & 0.071 & 0.091 & 0.111 & 0.041 & 0.044 & 0.062 \\
\hline & & 1500 & 0.104 & 0.119 & 0.151 & 0.065 & 0.064 & 0.090 \\
\hline & \multirow[t]{3}{*}{4 (power) } & 300 & 0.076 & 0.095 & 0.112 & 0.047 & 0.049 & 0.068 \\
\hline & & 700 & 0.100 & 0.107 & 0.135 & 0.060 & 0.053 & 0.087 \\
\hline & & 1500 & 0.139 & 0.156 & 0.201 & 0.086 & 0.085 & 0.130 \\
\hline & \multirow[t]{3}{*}{5 (power) } & 300 & 0.072 & 0.100 & 0.109 & 0.044 & 0.050 & 0.063 \\
\hline & & 700 & 0.129 & 0.137 & 0.177 & 0.074 & 0.084 & 0.110 \\
\hline & & 1500 & 0.228 & 0.295 & 0.310 & 0.165 & 0.205 & 0.225 \\
\hline \multirow{15}{*}{$\begin{array}{l}\gamma=0.10 \\
g=0.70\end{array}$} & \multirow[t]{3}{*}{1} & 300 & 0.047 & 0.093 & 0.071 & 0.026 & 0.047 & 0.038 \\
\hline & & 700 & 0.058 & 0.098 & 0.089 & 0.036 & 0.047 & 0.050 \\
\hline & & 1500 & 0.058 & 0.093 & 0.089 & 0.025 & 0.050 & 0.040 \\
\hline & \multirow[t]{3}{*}{2} & 300 & 0.064 & 0.099 & 0.093 & 0.035 & 0.045 & 0.052 \\
\hline & & 700 & 0.069 & 0.100 & 0.101 & 0.040 & 0.048 & 0.060 \\
\hline & & 1500 & 0.062 & 0.092 & 0.094 & 0.035 & 0.052 & 0.051 \\
\hline & \multirow[t]{3}{*}{3 (size) } & 300 & 0.087 & 0.103 & 0.120 & 0.055 & 0.052 & 0.073 \\
\hline & & 700 & 0.100 & 0.123 & 0.141 & 0.063 & 0.072 & 0.091 \\
\hline & & 1500 & 0.110 & 0.129 & 0.149 & 0.080 & 0.085 & 0.098 \\
\hline & \multirow[t]{3}{*}{4 (power) } & 300 & 0.129 & 0.128 & 0.172 & 0.086 & 0.068 & 0.115 \\
\hline & & 700 & 0.264 & 0.246 & 0.340 & 0.201 & 0.167 & 0.254 \\
\hline & & 1500 & 0.601 & 0.517 & 0.701 & 0.531 & 0.416 & 0.618 \\
\hline & \multirow[t]{3}{*}{5 (power) } & 300 & 0.193 & 0.214 & 0.249 & 0.141 & 0.141 & 0.182 \\
\hline & & 700 & 0.492 & 0.548 & 0.584 & 0.401 & 0.458 & 0.492 \\
\hline & & 1500 & 0.900 & 0.922 & 0.954 & 0.866 & 0.879 & 0.920 \\
\hline
\end{tabular}


Table 4: Relative Rejection Frequencies of the Tests for Null Hypothesis $H_{0}: r=3$ Based on Highly Persistent Five-Dimensional Processes

\begin{tabular}{|c|c|c|c|c|c|c|c|c|}
\hline \multirow{3}{*}{$\begin{array}{l}\text { GARCH } \\
\text { parameters }\end{array}$} & \multirow[b]{3}{*}{ true $r$} & \multirow[b]{3}{*}{$T$} & \multicolumn{6}{|c|}{ Nominal Size } \\
\hline & & & \multicolumn{3}{|c|}{$10 \%$} & \multicolumn{3}{|c|}{$5 \%$} \\
\hline & & & $L M(1)$ & $Q_{1}(1)$ & $\overline{Q_{2}(1)}$ & $L M(1)$ & $Q_{1}(1)$ & $Q_{2}(1)$ \\
\hline \multirow{15}{*}{$\begin{array}{l}\gamma=0.15 \\
g=0.75\end{array}$} & \multirow[t]{3}{*}{1} & 300 & 0.054 & 0.098 & 0.081 & 0.026 & 0.044 & 0.041 \\
\hline & & 700 & 0.060 & 0.088 & 0.082 & 0.033 & 0.044 & 0.048 \\
\hline & & 1500 & 0.059 & 0.101 & 0.088 & 0.031 & 0.052 & 0.050 \\
\hline & \multirow[t]{3}{*}{2} & 300 & 0.059 & 0.106 & 0.090 & 0.035 & 0.049 & 0.052 \\
\hline & & 700 & 0.067 & 0.104 & 0.104 & 0.038 & 0.051 & 0.057 \\
\hline & & 1500 & 0.061 & 0.092 & 0.095 & 0.035 & 0.040 & 0.049 \\
\hline & \multirow[t]{3}{*}{3 (size) } & 300 & 0.096 & 0.111 & 0.142 & 0.062 & 0.056 & 0.088 \\
\hline & & 700 & 0.093 & 0.114 & 0.131 & 0.059 & 0.065 & 0.088 \\
\hline & & 1500 & 0.078 & 0.108 & 0.106 & 0.051 & 0.065 & 0.068 \\
\hline & \multirow[t]{3}{*}{4 (power) } & 300 & 0.268 & 0.247 & 0.346 & 0.207 & 0.171 & 0.268 \\
\hline & & 700 & 0.690 & 0.605 & 0.769 & 0.619 & 0.512 & 0.700 \\
\hline & & 1500 & 0.965 & 0.930 & 0.987 & 0.947 & 0.897 & 0.978 \\
\hline & \multirow[t]{3}{*}{5 (power) } & 300 & 0.486 & 0.540 & 0.592 & 0.407 & 0.444 & 0.499 \\
\hline & & 700 & 0.917 & 0.947 & 0.965 & 0.893 & 0.909 & 0.947 \\
\hline & & 1500 & 0.996 & 1.000 & 1.000 & 0.995 & 0.999 & 1.000 \\
\hline \multirow{15}{*}{$\begin{array}{l}\gamma=0.17 \\
g=0.80\end{array}$} & \multirow[t]{3}{*}{1} & 300 & 0.051 & 0.107 & 0.078 & 0.024 & 0.057 & 0.035 \\
\hline & & 700 & 0.056 & 0.091 & 0.088 & 0.030 & 0.041 & 0.048 \\
\hline & & 1500 & 0.055 & 0.094 & 0.082 & 0.023 & 0.044 & 0.043 \\
\hline & \multirow[t]{3}{*}{2} & 300 & 0.061 & 0.097 & 0.092 & 0.030 & 0.046 & 0.049 \\
\hline & & 700 & 0.063 & 0.096 & 0.101 & 0.034 & 0.050 & 0.054 \\
\hline & & 1500 & 0.063 & 0.102 & 0.097 & 0.031 & 0.051 & 0.047 \\
\hline & \multirow[t]{3}{*}{3 (size) } & 300 & 0.094 & 0.110 & 0.138 & 0.063 & 0.061 & 0.088 \\
\hline & & 700 & 0.082 & 0.114 & 0.118 & 0.049 & 0.065 & 0.071 \\
\hline & & 1500 & 0.067 & 0.096 & 0.096 & 0.038 & 0.055 & 0.056 \\
\hline & \multirow[t]{3}{*}{4 (power) } & 300 & 0.421 & 0.390 & 0.510 & 0.348 & 0.305 & 0.440 \\
\hline & & 700 & 0.873 & 0.837 & 0.923 & 0.834 & 0.774 & 0.889 \\
\hline & & 1500 & 0.990 & 0.992 & 1.000 & 0.989 & 0.986 & 0.999 \\
\hline & \multirow[t]{3}{*}{5 (power) } & 300 & 0.725 & 0.746 & 0.814 & 0.674 & 0.660 & 0.752 \\
\hline & & 700 & 0.975 & 0.997 & 0.996 & 0.972 & 0.989 & 0.995 \\
\hline & & 1500 & 0.997 & 1.000 & 1.000 & 0.997 & 1.000 & 1.000 \\
\hline
\end{tabular}




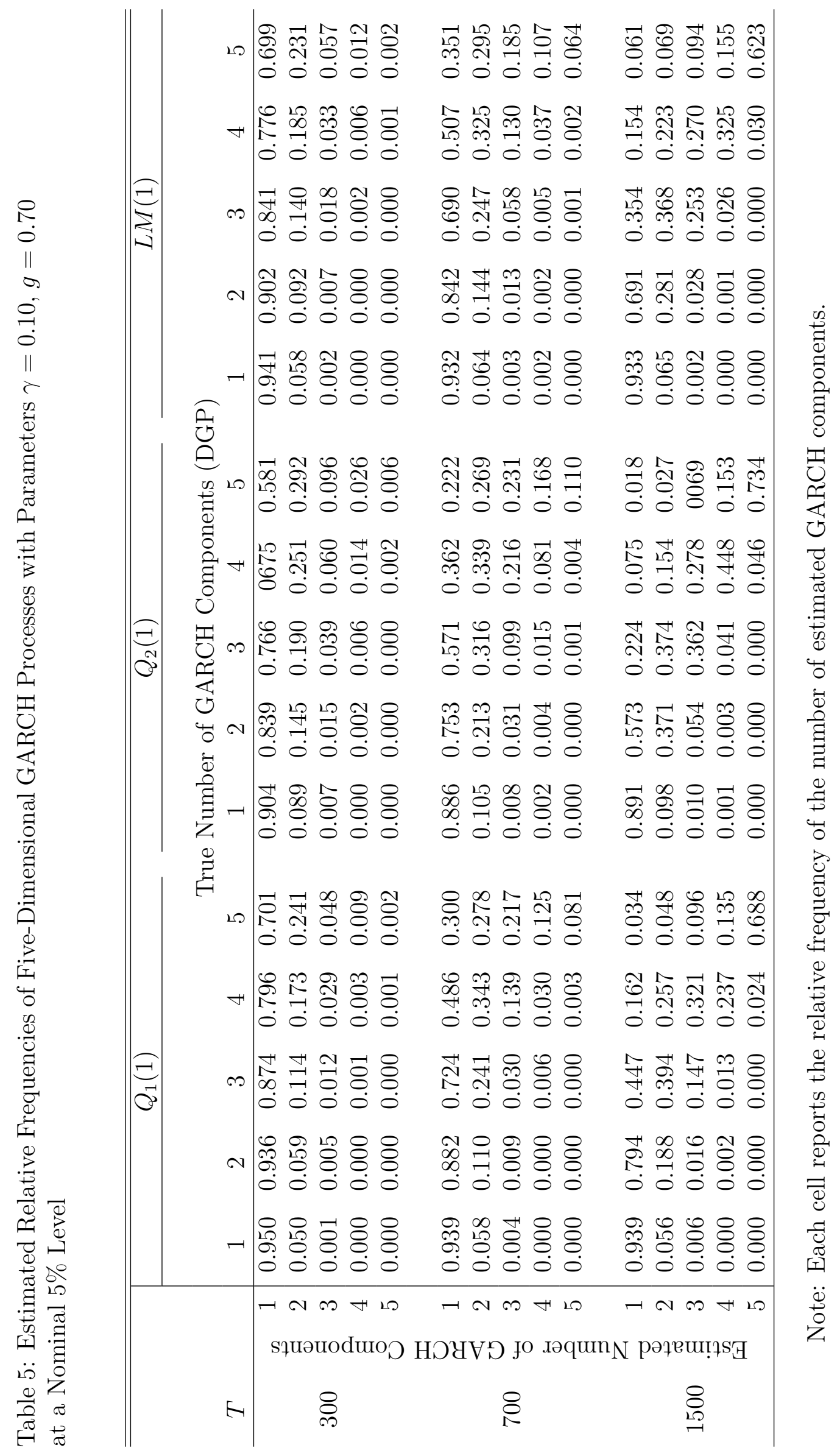




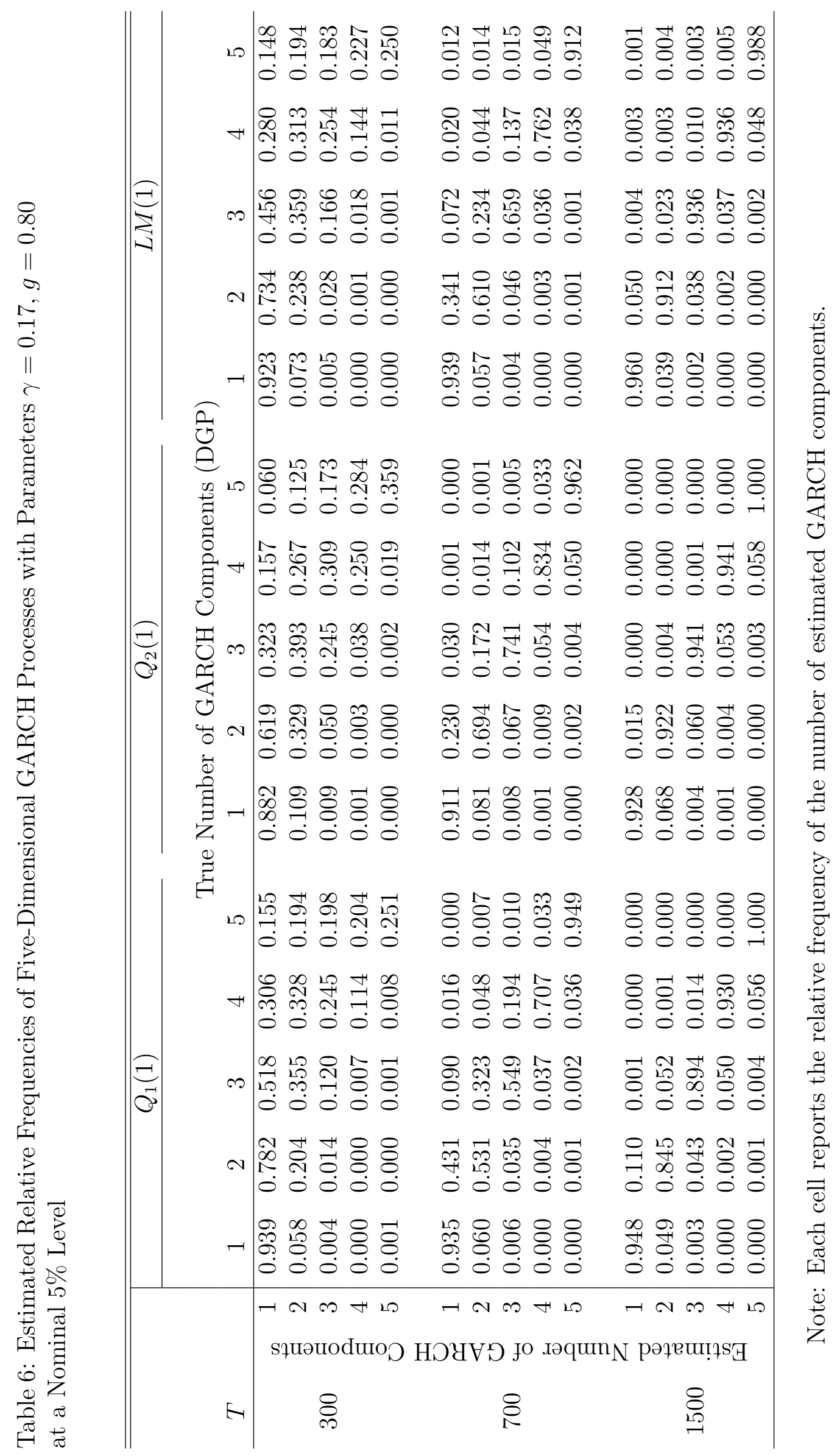


Table 7: $p$-Values for the Identification Tests Applied to the Data from Bouakez and Normandin (2010)

\begin{tabular}{cccccccc}
\hline \hline$H_{0}$ & Test & Canada & France & Germany & Italy & Japan & UK \\
\hline \multirow{5}{*}{$r=1$} & $L M(1)$ & 0.000 & 0.001 & 0.000 & 0.024 & 0.000 & 0.011 \\
& $Q_{1}(1)$ & 0.174 & 0.370 & 0.001 & 0.062 & 0.083 & 0.331 \\
& $Q_{2}(1)$ & 0.000 & 0.001 & 0.000 & 0.012 & 0.000 & 0.001 \\
& $\gamma_{1}+g_{1}$ & 0.986 & 0.983 & 0.983 & 0.990 & 0.990 & 0.989 \\
& & & & & & & \\
$r=2$ & $L M(1)$ & 0.027 & 0.014 & 0.000 & 0.037 & 0.000 & 0.007 \\
& $Q_{1}(1)$ & 0.183 & 0.457 & 0.005 & 0.049 & 0.000 & 0.311 \\
& $Q_{2}(1)$ & 0.024 & 0.010 & 0.000 & 0.020 & 0.000 & 0.000 \\
& $\gamma_{2}+g_{2}$ & 0.990 & 0.988 & 0.903 & 0.956 & 0.793 & 0.981 \\
& & & & & & & \\
& $L M(1)$ & 0.027 & 0.874 & 0.000 & 0.223 & 0.004 & 0.009 \\
$r=3$ & $Q_{1}(1)$ & 0.046 & 0.871 & 0.008 & 0.222 & 0.051 & 0.345 \\
& $Q_{2}(1)$ & 0.029 & 0.821 & 0.000 & 0.137 & 0.001 & 0.001 \\
& $\gamma_{3}+g_{3}$ & 0.792 & 0.671 & 0.814 & 0.882 & 0.984 & 0.528 \\
& & & & & & & \\
& $L M(1)$ & 0.150 & 0.673 & 0.679 & 0.696 & 0.025 & 0.104 \\
& $Q_{1}(1)$ & 0.139 & 0.467 & 0.412 & 0.353 & 0.050 & 0.180 \\
& $Q_{2}(1)$ & 0.127 & 0.581 & 0.650 & 0.501 & 0.019 & 0.012 \\
& $\gamma_{4}+g_{4}$ & 0.930 & 0.855 & 0.990 & 0.728 & 0.857 & 0.683 \\
& & & & & & & \\
& $L M(1)$ & 0.390 & 0.948 & 0.475 & 0.665 & 0.008 & 0.429 \\
& $Q_{1}(1)$ & 0.214 & 0.675 & 0.146 & 0.924 & 0.018 & 0.198 \\
& $Q_{2}(1)$ & 0.335 & 0.917 & 0.447 & 0.607 & 0.006 & 0.088 \\
& $\gamma_{5}+g_{5}$ & 0.207 & 0.912 & 0.963 & 0.985 & 0.952 & 0.984 \\
& & & & & & & \\
& $L M(1)$ & 0.689 & 0.751 & 0.380 & 0.511 & 0.057 & 0.714 \\
& $Q_{1}(1)$ & 0.617 & 0.712 & 0.105 & 0.320 & 0.047 & 0.161 \\
& $Q_{2}(1)$ & 0.632 & 0.669 & 0.348 & 0.455 & 0.051 & 0.607 \\
& $\gamma_{6}+g_{6}$ & 0.971 & 0.739 & 0.746 & 0.319 & 0.990 & 0.990 \\
& $Q_{7}+g_{7}$ & 0.194 & 0.900 & 0.392 & 0.579 & 0.246 & 0.363 \\
\hline & & & & & & & \\
& & & & & & &
\end{tabular}




\section{SFB 649 Discussion Paper Series 2015}

For a complete list of Discussion Papers published by the SFB 649, please visit http://sfb649.wiwi.hu-berlin.de.

001 "Pricing Kernel Modeling" by Denis Belomestny, Shujie Ma and Wolfgang Karl Härdle, January 2015.

002 "Estimating the Value of Urban Green Space: A hedonic Pricing Analysis of the Housing Market in Cologne, Germany" by Jens Kolbe and Henry Wüstemann, January 2015.

003 "Identifying Berlin's land value map using Adaptive Weights Smoothing" by Jens Kolbe, Rainer Schulz, Martin Wersing and Axel Werwatz, January 2015.

004 "Efficiency of Wind Power Production and its Determinants" by Simone Pieralli, Matthias Ritter and Martin Odening, January 2015.

005 "Distillation of News Flow into Analysis of Stock Reactions" by Junni L. Zhang, Wolfgang K. Härdle, Cathy Y. Chen and Elisabeth Bommes, January 2015.

006 "Cognitive Bubbles" by Ciril Bosch-Rosay, Thomas Meissnerz and Antoni Bosch-Domènech, February 2015.

007 "Stochastic Population Analysis: A Functional Data Approach" by Lei Fang and Wolfgang K. Härdle, February 2015.

008 "Nonparametric change-point analysis of volatility" by Markus Bibinger, Moritz Jirak and Mathias Vetter, February 2015.

009 "From Galloping Inflation to Price Stability in Steps: Israel 1985-2013" by Rafi Melnick and till Strohsal, February 2015.

010 "Estimation of NAIRU with Inflation Expectation Data" by Wei Cui, Wolfgang K. Härdle and Weining Wang, February 2015.

011 "Competitors In Merger Control: Shall They Be Merely Heard Or Also Listened To?" by Thomas Giebe and Miyu Lee, February 2015.

012 "The Impact of Credit Default Swap Trading on Loan Syndication" by Daniel Streitz, March 2015.

013 "Pitfalls and Perils of Financial Innovation: The Use of CDS by Corporate Bond Funds" by Tim Adam and Andre Guettler, March 2015.

014 "Generalized Exogenous Processes in DSGE: A Bayesian Approach" by Alexander Meyer-Gohde and Daniel Neuhoff, March 2015.

015 "Structural Vector Autoregressions with Heteroskedasticy" by Helmut Lütkepohl and Aleksei Netšunajev, March 2015.

016 "Testing Missing at Random using Instrumental Variables" by Christoph Breunig, March 2015.

017 "Loss Potential and Disclosures Related to Credit Derivatives - A CrossCountry Comparison of Corporate Bond Funds under U.S. and German Regulation" by Dominika Paula Gałkiewicz, March 2015.

018 "Manager Characteristics and Credit Derivative Use by U.S. Corporate Bond Funds" by Dominika Paula Gałkiewicz, March 2015.

019 "Measuring Connectedness of Euro Area Sovereign Risk" by Rebekka Gätjen Melanie Schienle, April 2015.

020 "Is There an Asymmetric Impact of Housing on Output?" by Tsung-Hsien Michael Lee and Wenjuan Chen, April 2015.

021 "Characterizing the Financial Cycle: Evidence from a Frequency Domain Analysis" by Till Strohsal, Christian R. Proaño and Jürgen Wolters, April 2015.

\section{SFB 649, Spandauer Straße 1, D-10178 Berlin http://sfb649.wiwi.hu-berlin.de}




\section{SFB 649 Discussion Paper Series 2015}

For a complete list of Discussion Papers published by the SFB 649, please visit http://sfb649.wiwi.hu-berlin.de.

022 "Risk Related Brain Regions Detected with 3D Image FPCA" by Ying Chen, Wolfgang K. Härdle, He Qiang and Piotr Majer, April 2015.

023 "An Adaptive Approach to Forecasting Three Key Macroeconomic Variables for Transitional China" by Linlin Niu, Xiu Xu and Ying Chen, April 2015.

024 "How Do Financial Cycles Interact? Evidence from the US and the UK" by Till Strohsal, Christian R. Proaño, Jürgen Wolters, April 2015.

025 "Employment Polarization and Immigrant Employment Opportunities" by Hanna Wielandt, April 2015.

026 "Forecasting volatility of wind power production" by Zhiwei Shen and Matthias Ritter, May 2015.

027 "The Information Content of Monetary Statistics for the Great Recession: Evidence from Germany" by Wenjuan Chen and Dieter Nautz, May 2015.

028 "The Time-Varying Degree of Inflation Expectations Anchoring" by Till Strohsal, Rafi Melnick and Dieter Nautz, May 2015.

029 "Change point and trend analyses of annual expectile curves of tropical storms" by P.Burdejova, W.K.Härdle, P.Kokoszka and Q.Xiong, May 2015.

030 "Testing for Identification in SVAR-GARCH Models" by Helmut Luetkepohl and George Milunovich, June 2015. 